\title{
Velocity and Density Effect on Impact Force during Water Entry of Sphere
}

Thomas Shepard, John Abraham*, Dillon Schwalbach, Seamus Kane, David Siglin and Teegan Harrington

School of Engineering, University of St. Thomas, 2115 Summit Avenue, St. Paul, MN 55105, USA

\begin{abstract}
A careful experimental investigation has been performed to quantify the impact forces on a sphere that passes from an air region to a water region. The experiments allowed changes to a wide range of parameters, including the impact velocity, the sphere density, and surface wettability. In addition to quantifying the impact force, results have allowed a qualitative discussion of the difference in fluid flow behaviour in the vicinity of the sphere. It was found that the parameters, particularly the sphere density, determined whether the adjoining air cavity would be quasi-static or deep-sealed. Non-dimensionalizing the input parameters reveals that there is a singular relationship which allows quantification of the average force coefficient up to the time of cavity pinch-off. Finally, it was found that the formation of quasi-static cavities resulted in larger impact forces compared to the deep-seal cases. The difference in these force coefficients did not depend on the dimensionless parameter.
\end{abstract}

Keywords: Water impact; Oceanography; Expendable bathythermograph; Temperature sensing; Climate change; Ocean heat content; Sphere impact forces

\section{Introduction}

When solid objects transit from one fluid region to another (e.g. from air to water), impact forces are exerted on the object and complex flow phenomena occur. The quantification of impact forces and flow features has both academic and applied motivations. In particular, for naval vessels and projectiles, the motion of objects which land on a free water surface or pass through the interface depend greatly on impact forces. Another important application is associated with the delivery of oceanographic measurement devices. Often, these devices are dropped into the ocean waters from varying heights above the surface. Knowledge of the speed of their decent and their trajectory is required for correct interpretation of measured data.

To be more specific regarding climatic sensing applications, the bulk of historical oceanographic temperature measurements have been made by probes such as the expendable bathythermograph [1]. For devices such as these, the depth is not a measured quantity; rather it is inferred from a calibrated fall rate equation which is developed during controlled experiments. It has been known that the actual depth depends on a variety of factors such as probe shape and rate of rotation [2-4], drop height and water temperature [5] or on other factors [6-7].

Despite an intense effort to fully quantify the descending motion of oceanographic sensing probes, adequate quantification of impact forces remains elusive. As a consequence, fully submerged modelling efforts do not encompass of all the relevant physical phenomena.

In order to deal with the lingering issue of impact forces, a series of studies has been carried out with simulation and experimentation used in tandom for the sphere entry problem [8-10]. Those studies have successfully matched the results from the two investigation methods (experimentation and computer simulation). Those studies also have identified the principle features of the flow field in the vicinity of the object. In addition, the effects of impact velocity, sphere density, and surface wettability have been studied. It was found that there is a correlation between the ratio of the kinetic energy of the sphere and the surface tension of the water with a dimensionless depth at which the sphere-induced liquid cavity closes. Furthermore, a dimensionless time for cavity closure was found to be constant and independent of sphere density, water impact, and surface waettability. Finally, the dimensionless time for cavity closure increased with the liquid Weber number.

Prior works [8-10] contain an extensive literature review which, for brevity will not be repeated here however interested readers are directed there. Among the prior works, [11] evaluated the impact forces on spinning objects and presented results in a dimensionless format. A similar approach is taken here.

In this work, the influence of sphere density and impact velocity on the net upward force, the dynamics of cavity formation, and the pinchoff of the cavity will be investigated. Also, the relationship between cavity shapes and impact force will be shown.

The sphere is the selected geometry of investigation because of its rich history of investigation. However, the methodologies and general conclusions can be extended to other blunt body shapes.

\section{Experimental Methods}

The water surface impact event was observed experimentally with the use of a high-speed digital camera system. A vacuum-release mechanism held the sphere at rest prior to release without imparting any forces on the sphere other than gravity at the moment of release. By varying the height of release, the impact velocity $\left(U_{o}\right)$ of the sphere was varied. The experimental set-up is illustrated in Figure 1.

As the sphere descended and impacted the water, images were captured at a rate of 5,400 frames per second using a Photron FASTCAM SA1.1 camera and a Nikon Nikkor 80-200 mm telephoto zoom lens. Three Lowell DP 1000W lights backlit the falling spheres to provide a clear contrast between the sphere and water for the purposes of image analysis. Each frame, at a resolution of 1024 x 1024 pixels,

*Corresponding author: John Abraham, School of Engineering, University of St Thomas, 2115 Summit Avenue, St. Paul, MN 55105, USA, Tel: 1-651-962-5766; E-mail: jpabraham@stthomas.edu

Received July 01, 2014; Accepted July 28, 2014; Published August 07, 2014

Citation: Shepard T, Abraham J, Schwalbach D, Kane S, Siglin D, et al. (2014) Velocity and Density Effect on Impact Force during Water Entry of Sphere. J Geophys Remote Sensing 3: 129. doi:10.4172/2169-0049.1000129

Copyright: @ 2014 Shepard T, et al. This is an open-access article distributed under the terms of the Creative Commons Attribution License, which permits unrestricted use, distribution, and reproduction in any medium, provided the original author and source are credited. 
Citation: Shepard T, Abraham J, Schwalbach D, Kane S, Siglin D, et al. (2014) Velocity and Density Effect on Impact Force during Water Entry of Sphere. J Geophys Remote Sensing 3: 129. doi:10.4172/2169-0049.1000129

Page 2 of 5
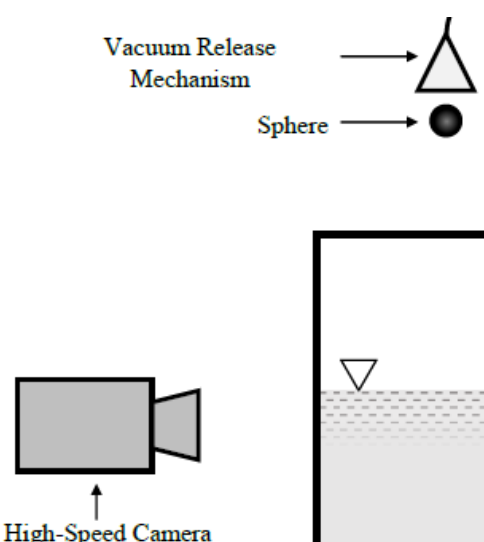

Water Tank

Figure 1: Experimental set-up.

\begin{tabular}{|c|c|c|c|}
\hline Variable & Value & Units & Uncertainty (\%) \\
\hline$\rho_{w}$ & 998 & $\mathrm{~kg} / \mathrm{m}^{3}$ & 1 \\
\hline$\mu$ & 0.001121 & $\mathrm{Ns} / \mathrm{m}^{2}$ & 2 \\
\hline$\sigma$ & 0.071 & $\mathrm{~N} / \mathrm{m}$ & 2 \\
\hline$\Theta$ & 77,115 & degrees & 15,5 \\
\hline$D$ & 3.962 & $\mathrm{~cm}$ & 0.3 \\
\hline$m$ & $21.33-220$ & $\mathrm{~g}$ & 0.3 \\
\hline$S G$ & $0.66-6.59$ & - & 1 \\
\hline$U_{o}$ & $2.6-6.5$ & $\mathrm{~m} / \mathrm{s}$ & 2 \\
\hline
\end{tabular}

Table 1: Experimental conditions.

provided a physical position of the sphere at a known time. The tank into which the spheres fell had dimensions of $27.94 \mathrm{~cm} \times 38.1 \mathrm{~cm} \times 76.2$ $\mathrm{cm}$ and was filled with tap water for the series of experiments.

To systematically vary the density of the sphere while holding the radius relatively constant, combinations of molten paraffin wax and steel shot were added to the interior volume of a hollow celluloid sphere through a hole which was patched after filling. The patched hole was subsequently sanded to match the contour of the sphere. Two spheres were constructed for each of the five sphere specific gravities examined ( $\mathrm{SG}=0.66-6.59$ ), with one left uncoated and one treated with a Rust-Oleum NeverWet hydrophobic surface coating.

The mass $(m)$ of each sphere was measured using a precision scale. Diameter $(D)$ measurements of each sphere were made using a micrometer. The surface contact angle $(\theta)$ of both the hydrophobic and non-treated spheres were measured using the sessile-drop method. The density $\left(\rho_{w}\right)$, viscosity $(\mu)$, and surface tension $(\sigma)$ properties of the tap water were measured using a hydrometer, capillary viscometer, and a DuNuoy tensiometer respectively. The experimental conditions of the experiments presented in this study are summarized in Table 1.

Image analysis of the captured frames was conducted using code developed with MATLAB. An intensity threshold was applied to each frame to isolate the sphere from the cavity and water. Then, the frame was converted to a binary image. The software extracted the position of the top-most and bottom-most pixels of the sphere. For the frames captured prior to cavity formation, the top-most pixel of the sphere was used to track the change in position of the sphere between frames. As the cavity began to form, the top-half of the sphere became obscured. Therefore, the bottom-most pixel of the sphere was used for position tracking once the cavity at that time. The pixel-to-meter scale was observably different between the air and water, so two different distance factors were applied as appropriate.

Once the position and time data were extracted from the captured image frames, the instantaneous velocity and acceleration of each sphere was determined using a quintic smoothing spline fit to the data. This fit allowed determination of the first and second derivatives of the curve to obtain velocity and acceleration, respectively. Readers interested in this method are directed to Epps, Truscott and Techet [12]. Repeated experiments indicated consistent results for each investigated set of conditions.

\section{Experimental Results}

The majority of experimental conditions in this study produced one of two distinct cavity types behind the sphere during entry. The hydrophobic sphere with a specific gravity of 0.66 formed a quasi-static cavity for all impact velocities examined. This cavity type is delineated by the cavity pinch-off location being extremely close to the sphere as shown in Figure 2. Very little air is left attached to the sphere in the quasi-static cavity seal. All spheres with a specific gravity of 1.05 6.66 formed a deep seal cavity regardless of surface coating or impact velocity. A deep seal cavity is formed when pinch-off occurred at some

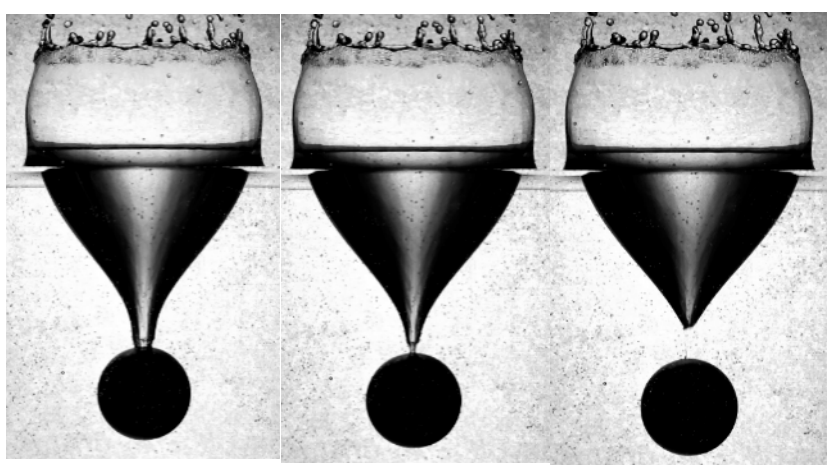

Figure 2: Quasi-static seal and pinch-off.

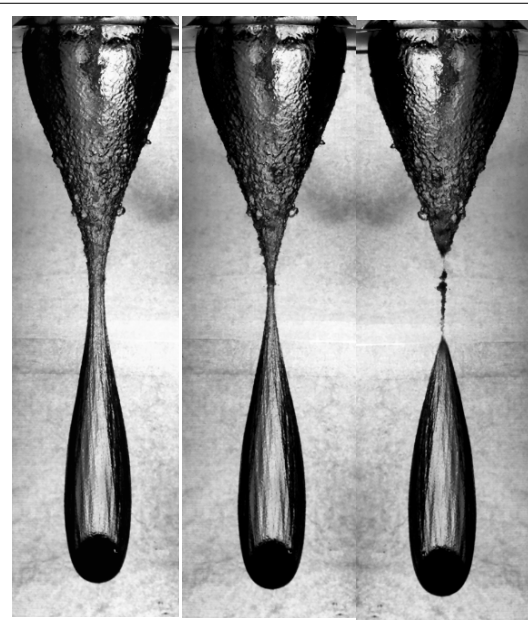

Figure 3: Deep-seal and pinch-off. 
intermediate distance between the sphere and free-surface as shown in Figure 3 .

Upon impact, the spheres experience a net upward force due to effects of momentum transfer to the water (added mass), surface tension, viscous drag and pressure forces. As discussed by Truscott, Epps and Techet [13], the pressure forces (buoyancy, added mass) dominate the upward force. The benefit of combining all hydrodynamic forces into a single time-varying term, $F(t)$, is that no assumptions are required regarding displaced water volume or cavity separation point from the sphere. Using the experimental data, the time-varying acceleration, $a(t)$ and velocity, $U(t)$, are determined and the total hydrodynamic force coefficient $\left(C_{f}\right)$ is arrived at as shown in Eqs. 1-3. Here, positive acceleration and forces are in the upward direction.

$$
\begin{aligned}
& m \cdot \vec{a}(t)=F(t)-m \cdot g \\
& F(t)=\frac{\pi}{8} \rho_{w} \cdot D^{2} \cdot[U(t)]^{2} C_{f} \\
& C_{f}=\frac{8 \cdot m(\vec{a}(t)+g)}{\pi \cdot \rho_{w} \cdot D^{2} \cdot[U(t)]^{2}}
\end{aligned}
$$

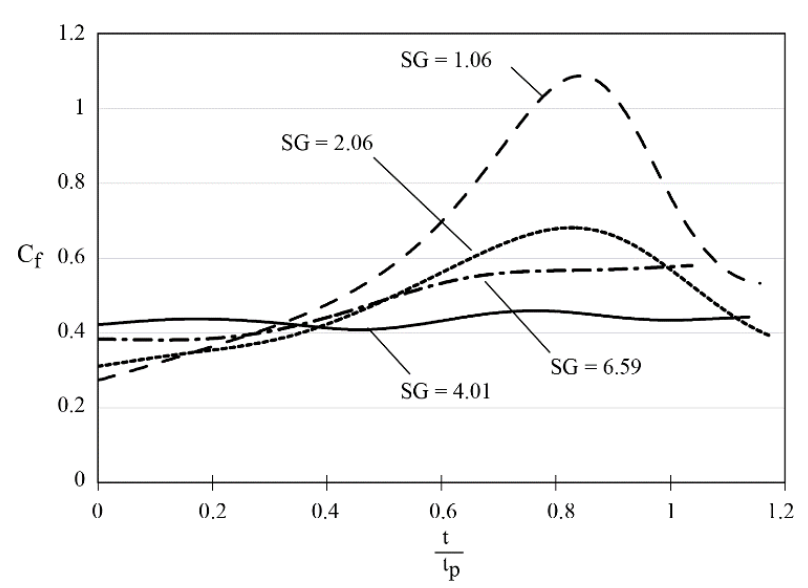

Figure 4: Effect of sphere specific gravity on force coefficient upon impact at $U_{0}=2.6 \mathrm{~m} / \mathrm{s}$ (deep-seal cavity).

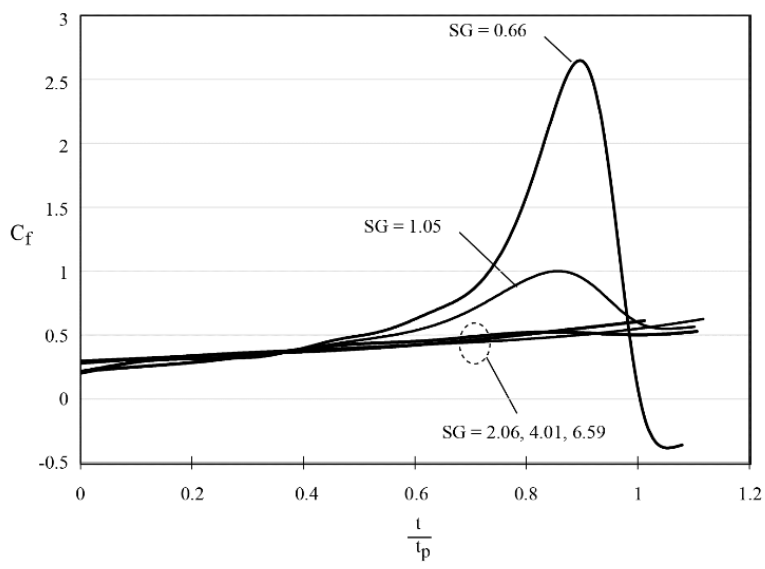

Figure 5: Effect of sphere specific gravity on force coefficient upon impact at $U_{0}=6.3 \mathrm{~m} / \mathrm{s}$ (deep-seal cavity).

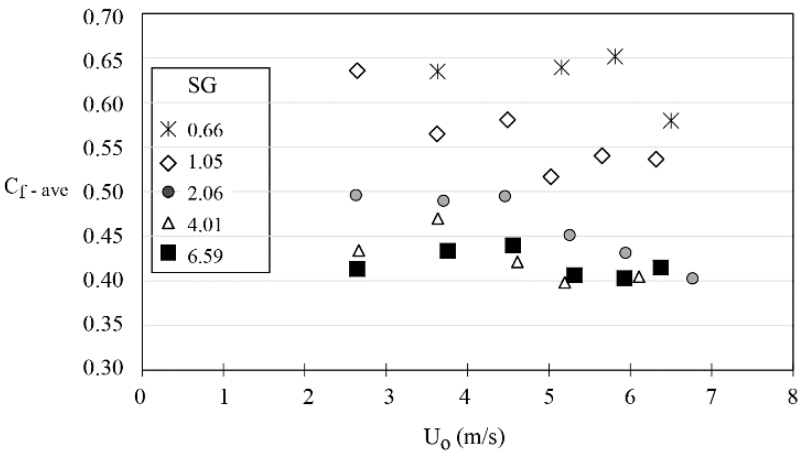

Figure 6: Effect of sphere specific gravity and impact velocity on timeaveraged force coefficient to pinch-off (deep-seal cavity).

The force coefficient is expected to be unsteady as the sphere impacts the water and the air cavity collapses. Resulting data will now be presented in the graphical form. Figures 4 and 5 show how the force coefficient depends on the specific gravity of the sphere and impact velocity for deep-seal cavity impacts. Figure 4 corresponds to a 2.6 $\mathrm{m} / \mathrm{s}$ impact velocity whereas Figure 5 is for $6.3 \mathrm{~m} / \mathrm{s}$. It is noted that time is measured from the instant the sphere contacts the free surface and results are made non-dimensional using the time at which cavity pinch-off occurs $\left(t_{p}\right)$. The data show that less dense spheres with low impact velocities result in a higher degree of unsteadiness as the cavity forms and collapses. As the cavity approaches pinch-off, the force coefficient can drop dramatically. This effect diminishes for sphere specific gravities above 2 and impact velocities of apporximately $5 \mathrm{~m} / \mathrm{s}$. In these cases, there is a much more linear and gradual increase in force coefficient during the transient deep-seal cavity dyanmics.

To demonstrate the effects of sphere specific gravity and impact velocity on the overall hydrodynamic force during the deep seal cavity dynamics, the force coefficient is averaged from the moment of impact to the moment of pinch-off $\left(C_{f \text { avg }}\right)$ as presented in Figure 6. There is a small trend showing that, on average, increasing the impact velocity results in a slight decrease in time-averaged force coefficient. The time averaged force coefficient also decreases with an increase in sphere specific gravity. As impact velocity increases above $5 \mathrm{~m} / \mathrm{s}$ and sphere specific gravity increases above 2 it is noted that there is very little variation in the average force coefficient.

Non-dimensionalization of the effects of sphere density, as captured in the specific gravity and impact velocity effectively results in a non-dimensional impact-momentum term. The effect of this term on the time-averaged force coefficient for deep seal cavities is shown in Figure 7 . The data shows a non-linearly decreasing trend which flattens out as impact momentum increases. A correlation of the data reveals the following relationship:

A comparison of the experimental data with Eq. 4 shows an average deviation of $\pm 6 \%$. To the best knowledge of the authors, this finding has not been found in the literature.

Experiments conducted on the $\mathrm{SG}=0.66$ spheres having different surface coatings resulted in different cavity types, thus allowing an examination of the effect that cavity type has on forces during water entry. Figure 8 shows the quasi-static (8A) and deep seal (8B) cavity pinching off. Though the cavity shapes above the pinch-off point look similar for either case, the air in contact with the sphere is quite 
Citation: Shepard T, Abraham J, Schwalbach D, Kane S, Siglin D, et al. (2014) Velocity and Density Effect on Impact Force during Water Entry of Sphere. J Geophys Remote Sensing 3: 129. doi:10.4172/2169-0049.1000129

Page 4 of 5

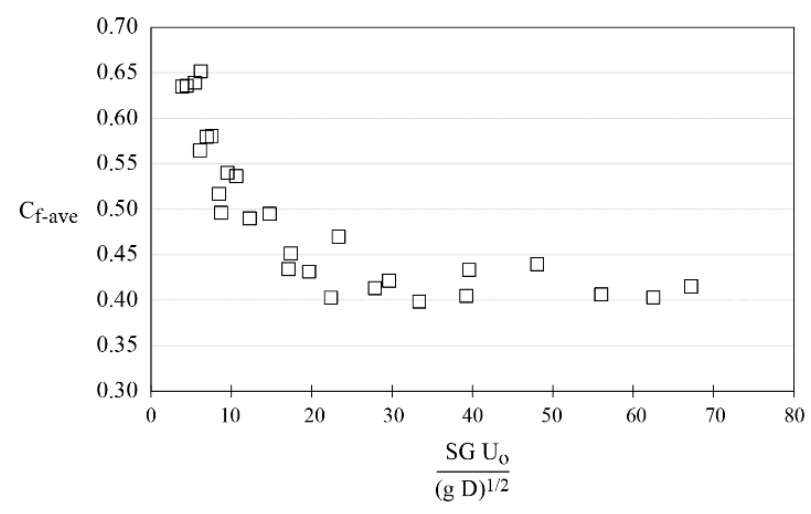

Figure 7: Effect of non-dimensional impact momentum on time-averaged force coefficient to pinch-off (deep-seal cavity).
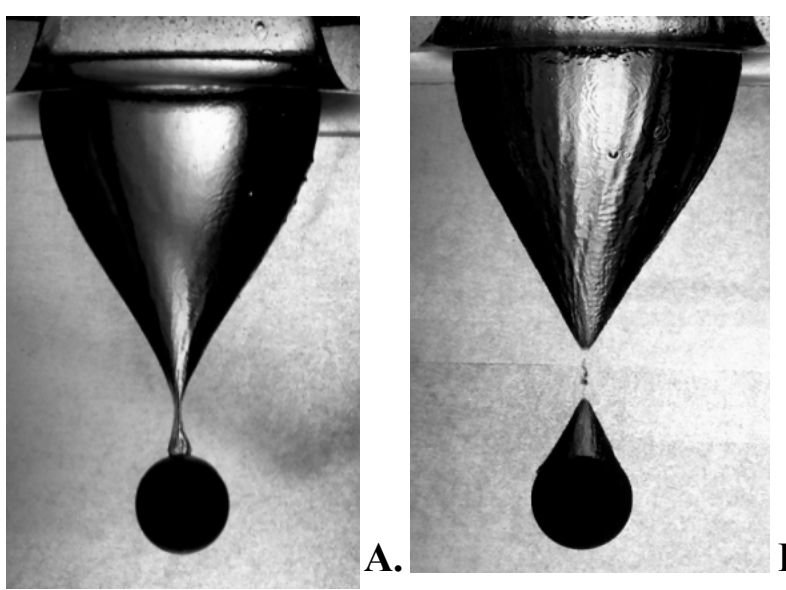

B.

Figure 8: Different seals for identical specific gravity (0.66) and impact velocity $(6.3 \mathrm{~m} / \mathrm{s})$ caused by surface treatment: A) Quasi-static seal at pinchoff, B) Deep-seal at pinch-off.

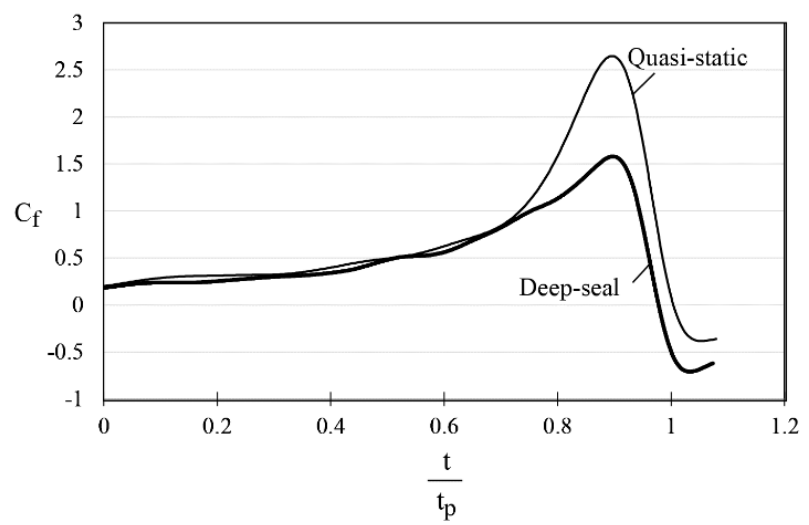

Figure 9: Effect of cavity type on force coefficient for $S G=0.66$ and $U_{0}=6.3$ $\mathrm{m} / \mathrm{s}$.

different. Figure 9 shows the effect that the different cavity types have on the unsteady force coefficient during impact. The impact coefficients follow very similar trends up to a dimensionless time of 0.75 . As the sphere descends past this time the area of contact between the air cavity and sphere starts to decrease for the quasi-static cavity. The maximum force coefficient is shown to be significantly higher for quasi-static

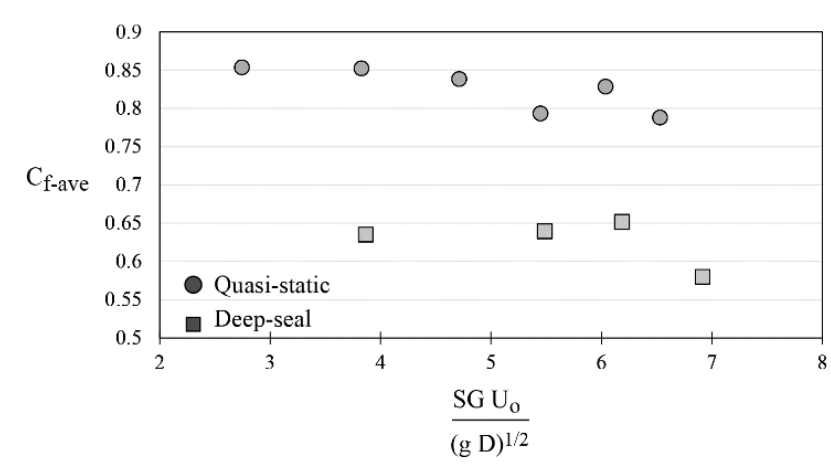

Figure 10: Effect of cavity type and non dimensional impact momentum on time-averaged force coefficient to pinch-off $(S G=0.66)$.

cavity in comparison to a deep-seal cavity. It is believed that the difference in impact force is associated with the larger co-moving water mass for the quasi-static case-the quasi-static case has a larger added mass of water. Here, the phrase added mass refers to the water which moves vertically along with the sphere.

The effect of cavity type on impact forces is further revealed in Figure 10. These data cover impact velocities of 2.3-6.5 m/s for the $\mathrm{SG}=0.66$ sphere. For the quasi-static seal the time-averaged impact force coefficient are $32 \%$ higher than those of the deep seal cavities on average. The results are largely independent of the dimensionless impact variable.

If one considers the quasi-static cavity as an intermediate case between no cavity conditions and deep-seal conditions, these results can be related to those of [13] in which it was shown that spheres which produce no cavity upon impact have higher force coefficients than cavity-forming cases. Truscott, Epps and Techet provide evidence that this result relates to differences in unsteady vortex formation. Additionally, it is noted in [14] that cavity closure events are accompanied by pressure pulses. As the two cavity cases produced in this study result in different contact areas between the air and sphere during collapse one might expect the pressure pulse to affect the spheres differently.

\section{Conclusion}

In this study, high-speed video was used to determine the effects of impact velocity and sphere density on the forces acting on a sphere during its initial impact, cavity formation and cavity pinch-off phases of water entry. Experimental results reveal that lower impact velocity leads to higher average force coefficients. On the other hand, increasing impact velocity or sphere density results in lower force coefficients, though this trend is shown to flatten out. A comparison of impact forces for spheres which had identical densities and impact velocities revealed that the type of cavity which forms affects the net hydrodynamic force. A cavity which experienced a deep-seal was shown to result in lower forces during water entry than quasi-static seal cavities.

\section{Acknowledgement}

The authors want to acknowledge the assistance of Dr. Brenden Epps in facilitating the use of the critical error determination of the best-fit splines. The high-speed imaging equipment used during this research was supported by the National Science Foundation under Grant No. 1229247.

\section{References}

1. Abraham JP, Baringer M, Bindoff NL, Boyer T, Cheng LJ, et al. (2013) A review 
Citation: Shepard T, Abraham J, Schwalbach D, Kane S, Siglin D, et al. (2014) Velocity and Density Effect on Impact Force during Water Entry of Sphere. J Geophys Remote Sensing 3: 129. doi:10.4172/2169-0049.1000129

Page 5 of 5

of global ocean temperature observations: implications for ocean heat content estimates and climate change. Reviews of Geophysics 51: 450-483.

2. Stark JR, Gorman JM, Hennessey MP, Reseghetti F, Willis JK, et al., (2011) A computational method for determining XBT depths. Ocean Sciences 7: 733743 .

3. Abraham JP, Gorman JM, Reseghetti F, Trenberth KE, Minkowycz WJ (2011) A new method of calculating ocean temperatures using expendable bathythermographs. Energy and Environment Research 1: 2-11.

4. Abraham JP, Gorman JM, Reseghetti F, Minkowycz WJ, Sparrow EM (2012) Turbulent and transitional modeling of drag on oceanographic measurement devices, Computational Fluid Dynamics and its Applications. Modelling and Simulation in Engineering.

5. Abraham JP, Gorman JM, Reseghetti F, Sparrow EM, Minkowycz WJ (2012) Drag coefficients for rotating expendable bathythermographs and the impact of launch parameters on depth predictions. Numerical Heat Transfer A 62: 25-43.

6. Cowley R, Wijffels S, Cheng L, Boyer T, Kizu S (2013) Biases in Expendable Bathythermograph Data: A New View Based on Historical Side-by-Side Comparisons Rebecca Cowley and Susan Wijfels. Journal of Atmospheric and Oceanic Technology 30: 1195-1225.

7. Ching L, Zhu J (2014) Uncertainties of the ocean heat content estimation induced by insufficient vertical resolution of historical ocean subsurface observations. Journal of Atmospheric and Oceanic Technology 31: 1383-1396.

8. Abraham JP, Gorman JM, Reseghetti F, Sparrow EM, Stark JR, et al. (2014) Modeling and numerical simulation of the forces action on a sphere during early-water entry. Ocean Engineering 76: 1-9.

9. Gorman JM, Abraham JP, Schwalbach DB, Shepard TG, Stark JR, et al. (2014) Experimental verification of drag forces on spherical objects entering water. $J$ Marine Biol and Oceanogr 3: 2.

10. Schwalbach DB, Shepard TG, Kane S, Siglin D, Harrington T, et al. (2014) Effect of impact velocity and mass ratio during vertical sphere water entry.

11. Truscott TT, Techet A (2009) Water entry of spinning spheres. J Fluid Mechanics 625: 1-31.

12. Epps BP, Truscott TT, Techet A (2010) Evaluating derivatives of experimental data using smoothing splines. Proceedings on Mathematical Methods in Engineering International Symposium.

13. Truscott TT, Epps BP, Techet A (2012) Unsteady forces on spheres during free-surface water entry. Journal of Fluid Mechanics 704: 173-210.

14. Abelson $\mathrm{HI}$ (1970) Pressure measurements in the water-entry cavity. Journal of Fluid Mechanics 44: 129-144. 